Research Article

\title{
Circuit Implementation Synchronization between Two Modified Fractional-Order Lorenz Chaotic Systems via a Linear Resistor and Fractional-Order Capacitor in Parallel Coupling
}

\author{
Juan Liu, ${ }^{1,2,3}$ Xuefeng Cheng, ${ }^{3,4}$ and Ping Zhou $\mathbb{D}^{4}$ \\ ${ }^{1}$ Chongqing University, Chongqing 400044, China \\ ${ }^{2}$ Chongqing Institute of Green and Intelligent Technology, Chinese Academy of Sciences, Chongqing 400714, China \\ ${ }^{3}$ Chongqing College, University of Chinese Academy of Sciences, Chongqing 400722, China \\ ${ }^{4}$ School of Science, Chongqing University of Posts and Telecommunications, Chongqing 400065, China
}

Correspondence should be addressed to Ping Zhou; zhouping@cqupt.edu.cn

Received 12 July 2021; Accepted 10 August 2021; Published 19 August 2021

Academic Editor: Viet-Thanh Pham

Copyright ( $\odot 2021$ Juan Liu et al. This is an open access article distributed under the Creative Commons Attribution License, which permits unrestricted use, distribution, and reproduction in any medium, provided the original work is properly cited.

\begin{abstract}
In this study, a modified fractional-order Lorenz chaotic system is proposed, and the chaotic attractors are obtained. Meanwhile, we construct one electronic circuit to realize the modified fractional-order Lorenz chaotic system. Most importantly, using a linear resistor and a fractional-order capacitor in parallel coupling, we suggested one chaos synchronization scheme for this modified fractional-order Lorenz chaotic system. The electronic circuit of chaos synchronization for modified fractional-order Lorenz chaotic has been given. The simulation results verify that synchronization scheme is viable.
\end{abstract}

\section{Introduction}

In the last twenty years, many fractional-order systems [1-9] have been used to discuss the dynamics, wave stability, initials, and boundary effect. Many real-world physical systems can be more accurately described by fractional-order differential equations (FODE) $[3-6,10,11]$, e.g., diffusion-wave, super diffusion, heat conduction, dielectric polarization, viscoelasticity, and electromagnetic waves. The complex behavior such as chaos has been observed in many physical fractionalorder systems, e.g., the fractional-order brushless DC motor chaotic system [6], the fractional-order Lorenz chaotic system [7], the fractional-order Chua's circuit [8], the fractional-order Duffing chaotic system [9], the fractional-order multistable locally active memristor [12], the fractional-order gyroscopes system [10], and the fractional-order microelectromechanical chaotic system [11]. Meanwhile, synchronization of chaotic systems has attracted extensive attention in recently years, and many synchronization strategies $[10,11,13-15]$ have been widely used in information processing, image encryption, network safety, secure communication, and machine learning.

On the other hand, many chaotic systems and synchronization strategies have been realized by electronic circuits [16-18]. So, synchronization between chaotic systems can be transferred to the synchronization between chaotic electronic circuits. Referring to synchronization between chaotic electronic circuits, the linear resistor coupling between two electronic circuits can realize the linear state variable coupling between chaotic systems, and the linear capacitive coupling or linear inductor coupling between two electronic circuits can realize the first derivative of state variable linear coupling. Up to now, many scholars [19-23] have proposed some synchronization approaches on integer-order chaotic electronic circuits by linear resistor coupling or linear capacitive coupling or a linear inductor coupling. However, to the best of our knowledge, there are little results on synchronization fractional-order chaotic electronic circuits coupled by a linear resistor or linear capacitive or linear inductor. Motivated by the 
abovementioned discussion, we have studied the modified fractional-order Lorenz chaotic system based on the integerorder Lorenz chaotic system. Furthermore, using a linear resistor and a fractional-order capacitor in parallel coupling, the chaos synchronization of modified fractional-order Lorenz chaotic electronic circuits has been achieved, while the linear resistor, capacitor, or inductor coupling is usually used in the existing works.

The rest of this study is as follows. In Section 2, a modified fractional-order Lorenz chaotic system is proposed, and its chaotic electronic circuits is designed. In Section 3, chaos synchronization between two modified fractional-order Lorenz chaotic systems is realized via a linear resistor and a fractional-order capacitor in parallel coupling, and the circuit experiment is obtained. Conclusions are drawn in Section 4.

\section{A Modified Fractional-Order Lorenz Chaotic System and Its Circuit Realization}

A modified Lorenz chaotic system [24] is described as follows:

$$
\left\{\begin{array}{l}
\frac{\mathrm{d} y_{1}}{\mathrm{~d} t}=40\left(y_{2}-y_{1}\right) \\
\frac{\mathrm{d} y_{2}}{\mathrm{~d} t}=10 y_{1}+25 y_{2}-y_{1} y_{3} \\
\frac{\mathrm{d} y_{3}}{\mathrm{~d} t}=y_{1} y_{2}-3 y_{3} .
\end{array}\right.
$$

Based on this integer-order Lorenz chaotic system (1), the modified fractional-order Lorenz chaotic system is obtained as follows:

$$
\left\{\begin{array}{l}
\frac{\mathrm{d}^{q} y_{1}}{\mathrm{~d} t}=40\left(y_{2}-y_{1}\right), \\
\frac{\mathrm{d}^{q} y_{2}}{\mathrm{~d} t}=10 y_{1}+25 y_{2}-y_{1} y_{3}, \\
\frac{\mathrm{d}^{q} y_{3}}{\mathrm{~d} t}=y_{1} y_{2}-3 y_{3},
\end{array}\right.
$$

where $q=0.95$ is the fractional order. Herein, the Caputo fractional-order derivatives are used. It is defined as

$$
\frac{\mathrm{d}^{q} f(t)}{\mathrm{d} t}=\frac{1}{\Gamma(1-q)} \int_{0}^{t} \frac{f^{\prime}(\tau)}{(t-\tau)^{q}} \mathrm{~d} \tau, \quad 0<q<1 .
$$

The Lyapunov exponents for this modified fractionalorder Lorenz chaotic system (2) can be calculated by the numerical method $[25,26]$, and the Lyapunov exponents are $(2.6245,-0.0461,-5.9568)$. The positive Lyapunov exponent indicates that chaotic attractors are emerged in system (2). Let $q=0.95$, and the chaotic attractors of the fractionalorder system (2) are shown in Figure 1.
Up to now, circuit implementation of chaotic systems has attracted more and more attentions. Many chaotic circuits have been reported. Now, we discuss the circuit implementation for the fractional-order chaotic system (2). Limited by the output of the operational amplifier, the amplitude of every variable in system (1) must be decreased, and we can choose $y_{1}=10 x_{1}, y_{2}=10 x_{2}$, and $y_{3}=10 x_{3}$. So, system (2) can be changed as the following system:

$$
\left\{\begin{array}{l}
\frac{\mathrm{d}^{q} x_{1}}{\mathrm{~d} t}=40\left(x_{2}-x_{1}\right), \\
\frac{\mathrm{d}^{q} x_{2}}{\mathrm{~d} t}=10 x_{1}+25 x_{2}-10 x_{1} x_{3}, \\
\frac{\mathrm{d}^{q} x_{3}}{\mathrm{~d} t}=10 x_{1} x_{2}-3 x_{3} .
\end{array}\right.
$$

Recently, the guidelines to design circuits for fractionalorder chaotic systems have been proposed in many studies [26-29]. To ordinary differential equation (ODE), we can use the linear capacitor $C$ to realize the first-order differential unit $\left(\mathrm{d} y_{i} / \mathrm{d} t\right), i=1,2,3$. Therefore, compared to the design of the first-order differential circuit unit in ODE, in order to realize the fractional-order differential unit $\left(\mathrm{d}^{q} x_{i} / \mathrm{d} t\right)$, $i=1,2,3$, we use the fractional-order capacitor $F$ replacing the linear capacitor $C$ in the first-order differential circuit unit in this study. According to [29], the fractional-order capacitor $F$ is shown as Figure 2, and it can realize the operator $\left(\mathrm{d}^{q} / \mathrm{d} t\right)$. In Figure $2, C_{1}=3.616 \mu \mathrm{F}, C_{2}=4.602 \mu \mathrm{F}$, $C_{3}=1.267 \mu \mathrm{F}, R_{a}=15.1 \mathrm{k} \Omega, R_{b}=1.51 \mathrm{M} \Omega, R_{c}=692.9 \mathrm{M} \Omega$.

Based on the circuitry design method for fractionalorder nonlinear systems in [25-29], the circuit diagram to realize the fractional-order nonlinear chaotic system (4) is presented as Figure 3.

In Figure 3, the operational amplifiers are the type of LF353N, the multipliers are the type of AD633, and the power is supplied by $\pm 15 \mathrm{~V}$, and $R_{1}=10 \mathrm{k} \Omega, R_{2}=2.5 \mathrm{k} \Omega$, $R_{3}=4 \mathrm{k} \Omega$, and $R_{4}=33.3 \mathrm{k} \Omega$. Using electronics workbench (EWB), we can obtain the chaotic attractors in the fractionalorder chaotic circuit, and the chaotic attractors are shown in Figure 4 .

According to the results in Figures 1 and 4, the fractional-order Lorenz chaotic system (4) has been circuit implementation as Figure 3.

\section{Synchronization of the Fractional-order Chaotic System (4) via a Linear Resistor and Fractional-Order Capacitor in Parallel Coupling}

In this section, synchronization of the chaotic system (4) is discussed. Let system (4) as the driving system. The response system is as follows: 


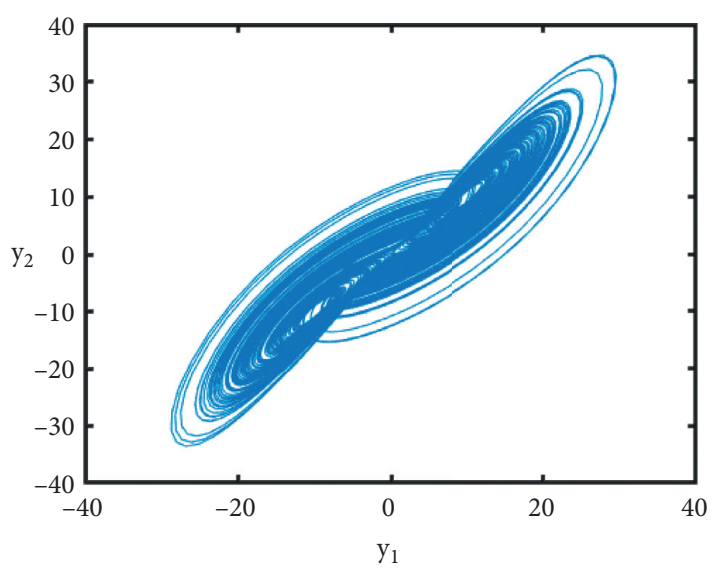

(a)

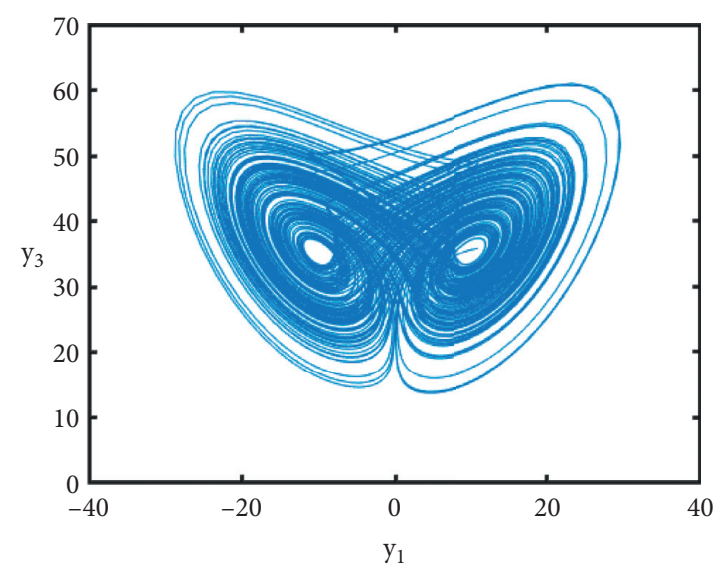

(b)

Figure 1: The chaotic attractors of the fractional-order system (2) for $q_{1}=q_{2}=q_{3}=0.95$. (a) The $y_{1} y_{2}$ phase diagram. (b) The $y_{1} y_{3}$ phase diagram.

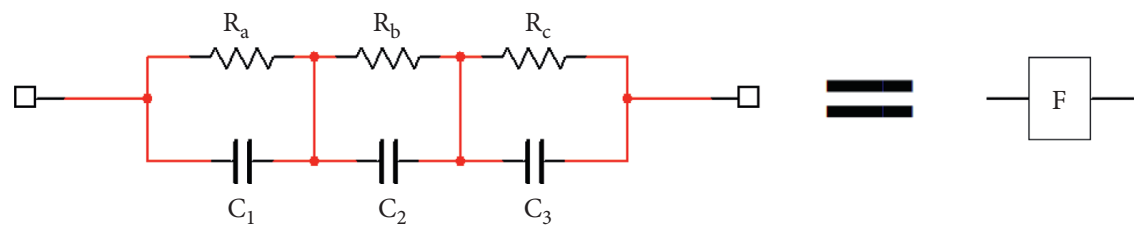

Figure 2: Circuit unit of the fractional-order capacitor $F$ for $q=0.95$.

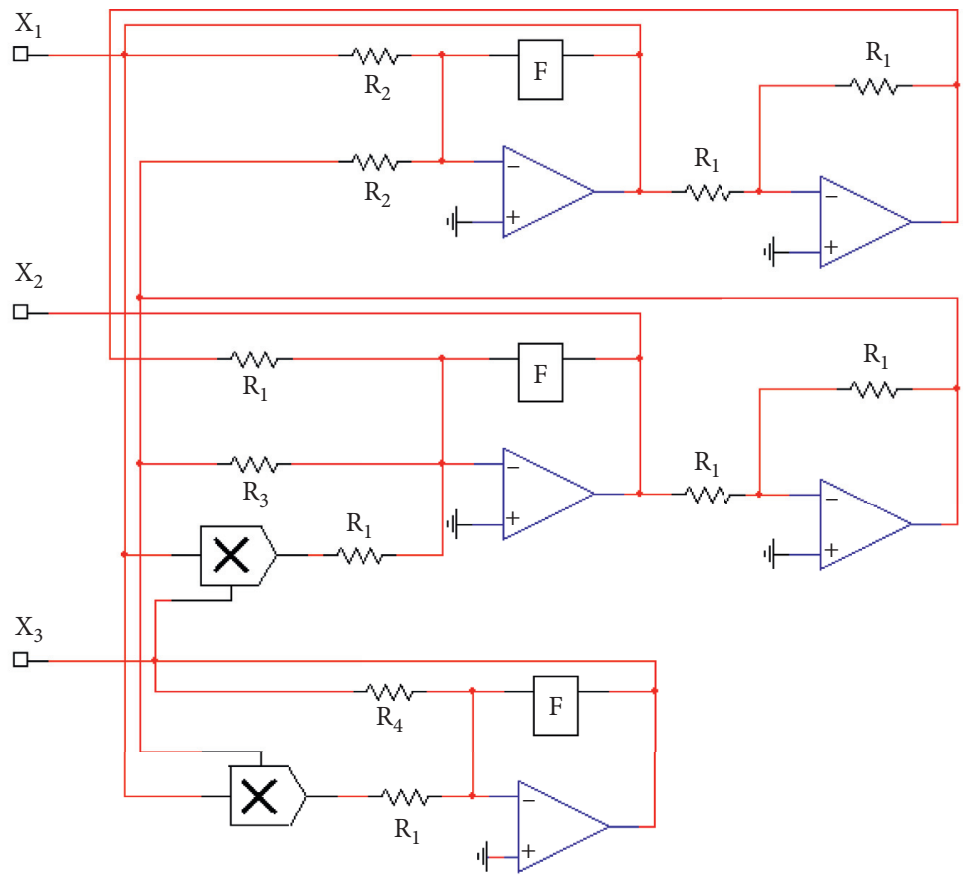

Figure 3: The circuit diagram to realize the fractional-order system (4) for $q=0.95$. 


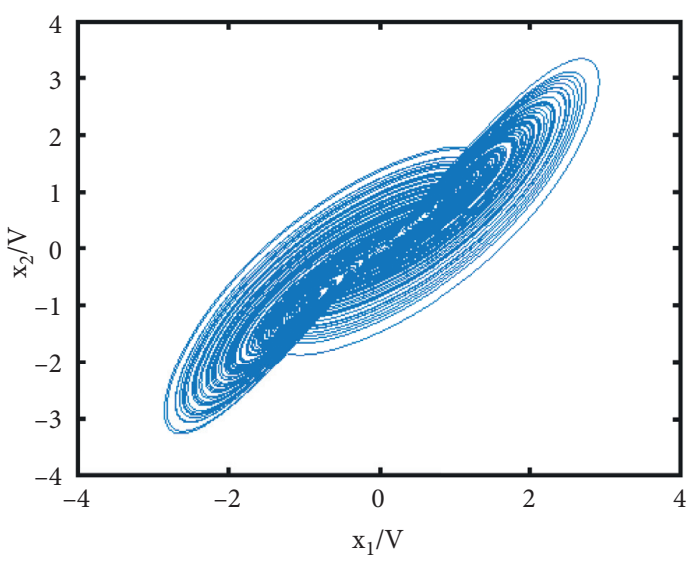

(a)

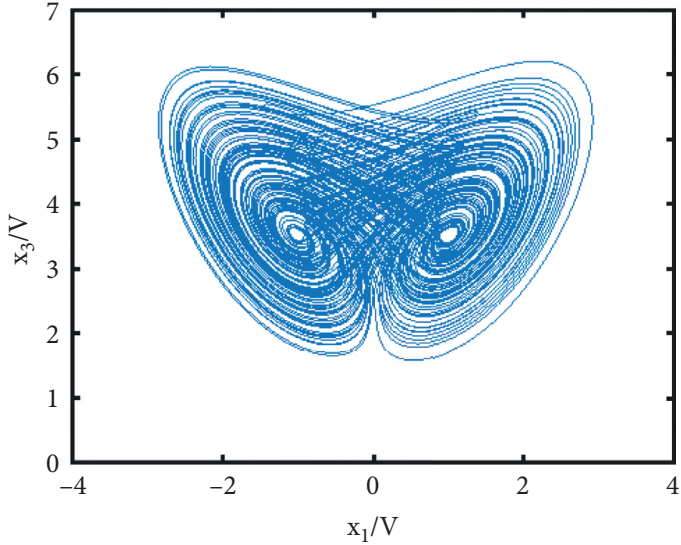

(b)

Figure 4: The circuit experiment phase portraits of the fractional-order chaotic system (4) for $q=0.95$. (a) The $x_{1} x_{2}$ phase diagram. (b) The $x_{1} x_{3}$ phase diagram.

$$
\left\{\begin{array}{l}
\frac{\mathrm{d}^{q} y_{1}}{\mathrm{~d} t}=40\left(y_{2}-y_{1}\right), \\
\frac{\mathrm{d}^{q} y_{2}}{\mathrm{~d} t}=10 y_{1}+25 y_{2}-10 y_{1} y_{3}, \\
\frac{\mathrm{d}^{q} y_{3}}{\mathrm{~d} t}=10 y_{1} y_{2}-3 y_{3} .
\end{array}\right.
$$

According to Section 2, circuit implementation of the fractional-order chaotic system (5) is the same as Figure 3. Now, we discuss how to realize the chaos synchronization between the drive system (4) and response system (5) via circuit implementation. In this study, the state variable $x_{2}$ in the driving system (4) and the state variable $y_{2}$ in the response system (5) are coupled via linear resistor $R_{k}$ and fractional-order capacitor $F$ in parallel, and its circuit diagram is shown in Figure 5. The green box in Figure 5 is the coupling part, and resistor $R_{5}=100 \mathrm{k} \Omega$.

According to Figure 5, one can obtain the following:

$$
u=K_{R}\left(x_{2}-y_{2}\right)+\left(\frac{\mathrm{d}^{q} x_{2}}{\mathrm{~d} t}-\frac{\mathrm{d}^{q} y_{2}}{\mathrm{~d} t}\right) .
$$

Here, variable $K_{R}=100 \mathrm{k} \Omega / R_{k}$. Now, according to Figure 5, the coupled driving system and coupled response system are shown as follows:

$$
\begin{aligned}
& \left\{\begin{array}{l}
\frac{\mathrm{d}^{q} x_{1}}{\mathrm{~d} t}=40\left(x_{2}-x_{1}\right), \\
\frac{\mathrm{d}^{q} x_{2}}{\mathrm{~d} t}=10 x_{1}+25 x_{2}-10 x_{1} x_{3}-K_{R}\left(x_{2}-y_{2}\right)-\left(\frac{\mathrm{d}^{q} x_{2}}{\mathrm{~d} t}-\frac{\mathrm{d}^{q} y_{2}}{\mathrm{~d} t}\right), \\
\frac{\mathrm{d}^{q} x_{3}}{\mathrm{~d} t}=10 x_{1} x_{2}-3 x_{3}, \\
\frac{\mathrm{d}^{q} y_{1}}{\mathrm{~d} t}=40\left(y_{2}-y_{1}\right), \\
\frac{\mathrm{d}^{q} y_{2}}{\mathrm{~d} t}=10 y_{1}+25 y_{2}-10 y_{1} y_{3}+K_{R}\left(x_{2}-y_{2}\right)+\left(\frac{\mathrm{d}^{q} x_{2}}{\mathrm{~d} t}-\frac{\mathrm{d}^{q} y_{2}}{\mathrm{~d} t}\right), \\
\frac{\mathrm{d}^{q} y_{3}}{\mathrm{~d} t}=10 y_{1} y_{2}-3 y_{3} .
\end{array}\right.
\end{aligned}
$$




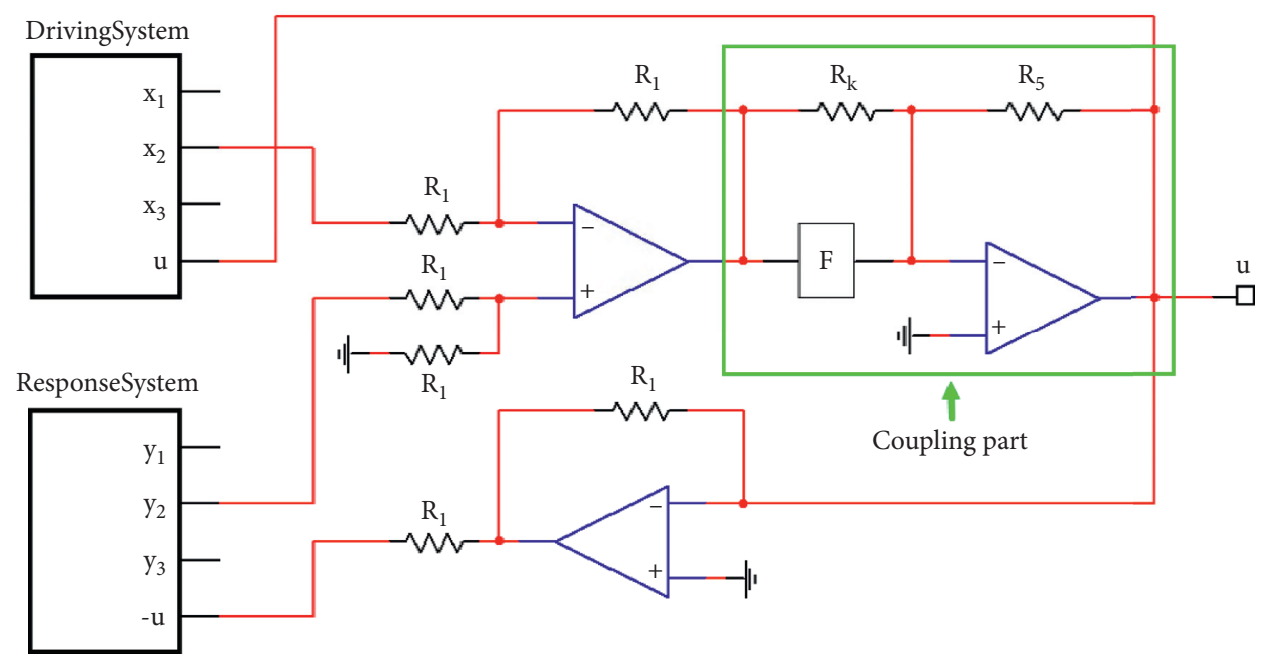

FIgURE 5: The circuit realization of chaos synchronization between the drive system (4) and response system (5).

Next, we discuss how to realize chaos synchronization between the drive system (7) and response system (8) via linear resistor $R_{k}$ or variable $K_{R}=100 \mathrm{k} \Omega / R_{k}$. Let synchronization errors $e_{i}=x_{i}-y_{i}(i=1,2,3)$, and we can obtain the error system as follows:

$$
\left\{\begin{array}{l}
\frac{\mathrm{d}^{q} e_{1}}{\mathrm{~d} t}=40\left(e_{2}-e_{1}\right), \\
\frac{\mathrm{d}^{q} e_{2}}{\mathrm{~d} t}=\frac{\left[10 e_{1}+\left(25-2 K_{R}\right) e_{2}+10 e_{1} e_{3}-10 e_{1} x_{3}-10 e_{3} x_{1}\right]}{3}, \\
\frac{\mathrm{d}^{q} e_{3}}{\mathrm{~d} t}=-3 e_{3}-10 e_{1} e_{2}+10 e_{1} x_{2}+10 e_{2} x_{1} .
\end{array}\right.
$$

According to the error system (9), $e_{i}=0(i=1,2,3)$ is the equilibrium point of the error system (9). If the equilibrium point $e_{i}=0(i=1,2,3)$ is asymptotic stability, then chaotic synchronization between the driving system (7) and response system (8) can be achieved. This result indicates that the synchronization of the fractional-order chaotic system (4) can be realized via the linear resistor $R_{k}$ and fractional-order capacitor $F$ in parallel coupling. On the other hand, if all the Lyapunov exponents (LEs) in system (9) are negative, then chaos synchronization is achieved. In this study, the Lyapunov exponents (LEs) are used to check the chaos synchronization.

Now, MATLAB is used to study the $\mathrm{QR}$ decomposition to analyze the synchronization problem of variable parameter $K_{R}$. The Jacobi matrix of the error system (9) is as follows:

$$
J=\left[\begin{array}{ccc}
-40 & 40 & 0 \\
\frac{\left(10+10 e_{3}-10 x_{3}\right)}{3} & \frac{\left(25-2 K_{R}\right)}{3} & \frac{\left(10 e_{1}-10 x_{1}\right)}{3} \\
-10 e_{2}+10 x_{2} & -10 e_{1}+10 x_{1} & 0
\end{array}\right] .
$$

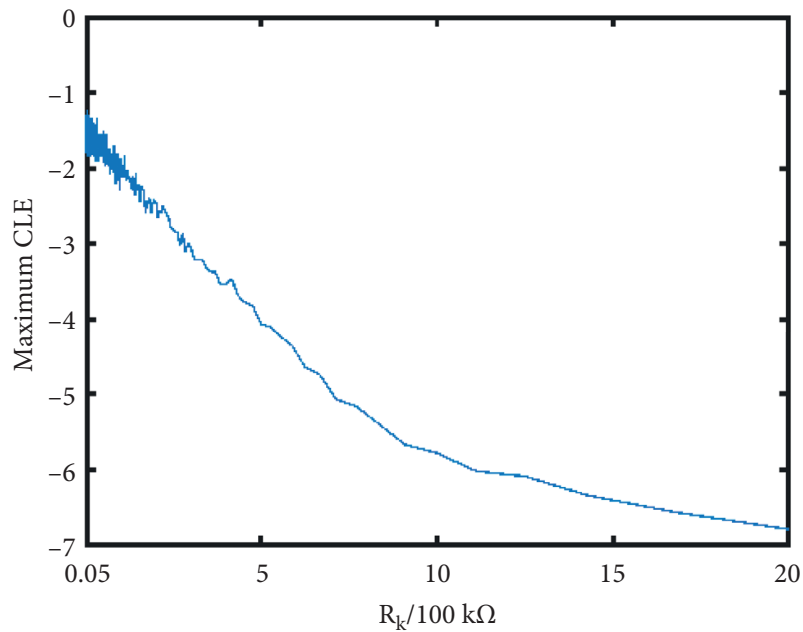

FIgURE 6: The maximum CLEs of error system (9) with variable $K_{R}=100 \mathrm{k} \Omega / R_{k}$.

The maximum Lyapunov exponents (CLEs) [24, 25] related to $R_{k}$ is shown in Figure 6. It can be seen that the maximum CLEs are negative if $K_{R}=0.05 \sim 20$. So, choosing linear resistor $R_{k}=(20 \sim 0.05) \times 100 \mathrm{k} \Omega$, the chaos synchronization between the drive system (7) and response system (8) can be obtained.

Choose $K_{R}=1$, then $R_{k}=100 \mathrm{k} \Omega$. We can yield that all the CLEs of the error system (9) are $\lambda_{1}=-2.4781$, $\lambda_{2}=-3.2007$, and $\lambda_{3}=-5.7004$. So, the chaos synchronization between the drive system (7) and response system (8) can be achieved. Take the initial driving signals $x=(2,2,2)$ and the initial response signals $y=(-2,-5,5)$, and the synchronization results are shown in Figure 7.

Choose $K_{R}=10$ and then $R_{k}=10 \mathrm{k} \Omega$. According to Figure 6, the maximum CLEs of the error system (9) is negative. So, the chaos synchronization between the drive system (7) and response system (8) can be achieved. Taking the initial driving 

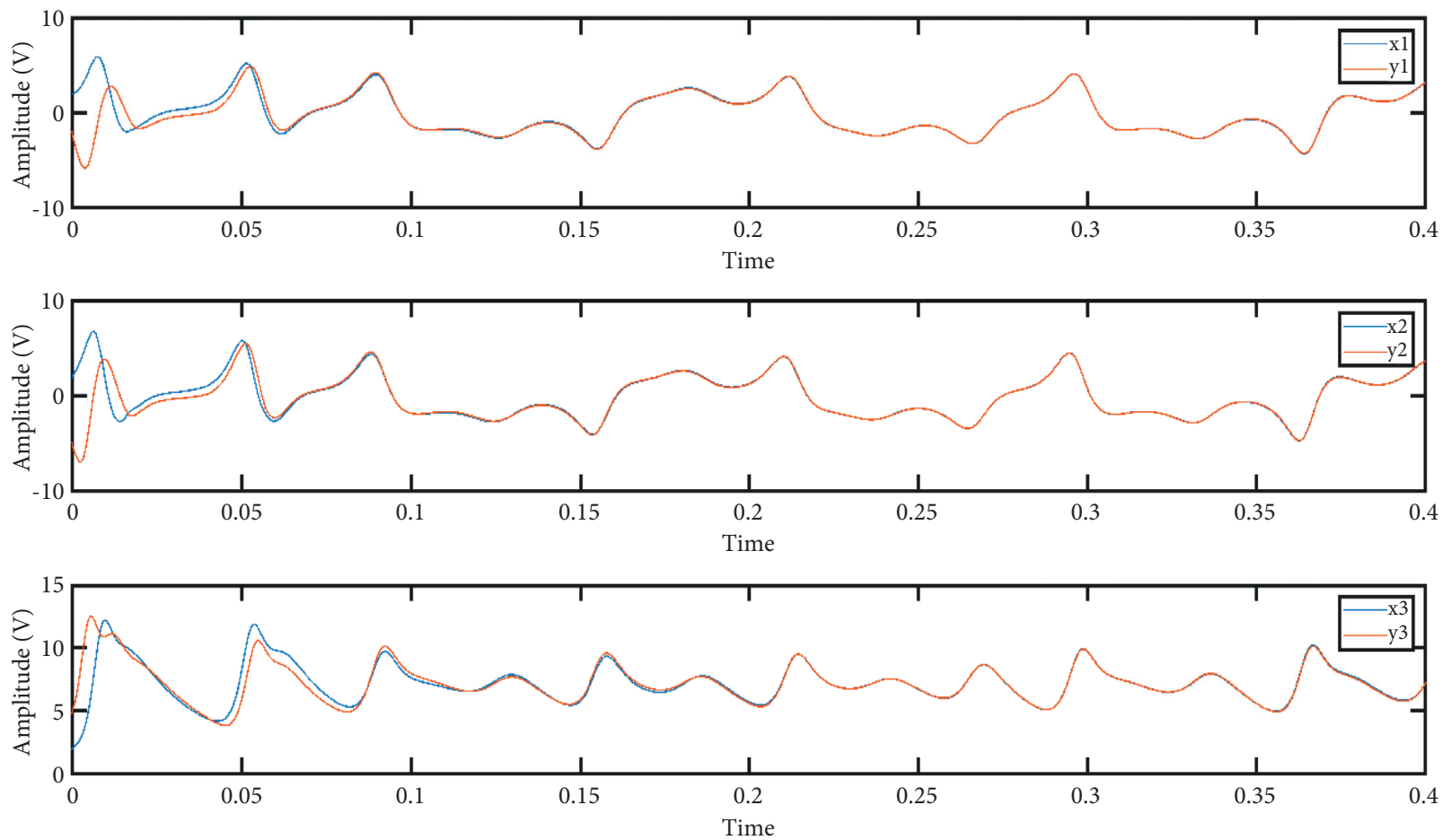

FIgURE 7: The synchronization process on $K_{R}=1$.
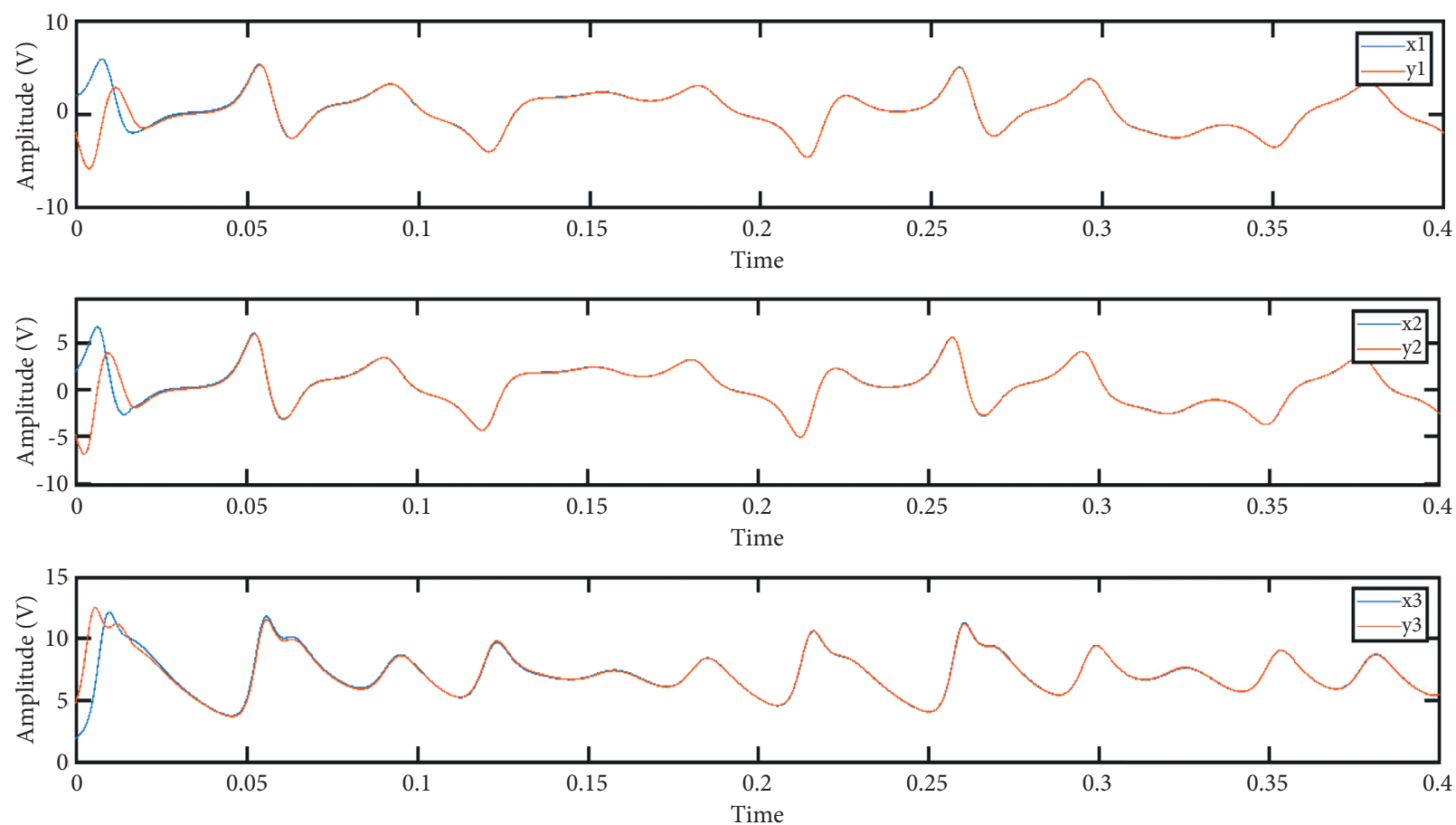

FIgURE 8: The synchronization process on $K_{R}=10$.

signals $x=(2,2,2)$ and the initial response signals $y=(-2,-5,5)$, the synchronization results are shown in Figure 8.

In summary, the chaos synchronization of the fractionalorder chaotic system (4) can be realized via the linear resistor $R_{k}$ and fractional-order capacitor $F$ in parallel coupling, and the maximum CLEs of the error system (9) with the linear resistor $R_{k}$ are obtained. Furthermore, we find that chaotic synchronization cannot be reached via state variable $x_{1}$ and $y_{1}$ coupling or via state variable $x_{3}$ and $y_{3}$ coupling. In addition, we find that chaotic synchronization can be arrived via only linear resistor coupling or via only fractional-order capacitor coupling. 


\section{Conclusions}

In this study, based on one modified Lorenz chaotic system, a modified fractional-order Lorenz chaotic system is suggested. We find that chaotic attractors are emerged in this modified fractional-order Lorenz chaotic system for $q=0.95$. Furthermore, we discussed the circuit implementation for this fractional-order chaotic system, and the circuit diagram to realize the fractional-order nonlinear chaotic system (4) is presented. More importantly, a synchronization scheme is suggested to realize the chaos synchronization on this modified fractional-order Lorenz chaotic system for $q=0.95$ via a linear resistor $R_{k}$ and a fractional-order capacitor $F$ in parallel coupling, which has not been used in the existing study. The electronic circuits for chaos synchronization of the modified fractional-order Lorenz chaotic system have been given. The simulation results verify that synchronization of the chaotic electronic circuit can be achieved. In the following study, whether inductive coupling can achieve synchronization is worth discussing.

\section{Data Availability}

The data used to support the findings of this study are available from the corresponding author upon request.

\section{Conflicts of Interest}

The authors declare that they have no conflicts of interest.

\section{Acknowledgments}

The authors thank Professor X. Luo for valuable suggestion.

\section{References}

[1] C. Li and G. Peng, "Chaos in Chen's system with a fractional order," Chaos, Solitons \& Fractals, vol. 22, no. 2, pp. 443-450, 2004.

[2] M. S. Tavazoei and M. Haeri, "A necessary condition for double scroll attractor existence in fractional-order systems," Physics Letters A, vol. 367, no. 1, pp. 102-113, 2007.

[3] R. Hilfer, Applications of Fractional Calculus in Physics, World Scientific, Singapore, 2000.

[4] I. Podlubny, Fractional Differential Equations, Academic Press, San Diego, CA, USA, 1999.

[5] P. Zhou, J. Ma, and J. Tang, "Clarify the physical process for fractional dynamical systems," Nonlinear Dynamics, vol. 100, no. 3, pp. 2353-2364, 2020.

[6] P. Zhou, R. J. Bai, and J. M. Zheng, "Stabilization of a fractional-order chaotic brushless DC motor via a single input," Nonlinear Dynamics, vol. 82, no. 1-2, pp. 519-525, 2015.

[7] I. Grigorenko and E. Grigorenko, "Chaotic dynamics of the fractional Lorenz system," Physical Review Letters, vol. 91, no. 3, pp. 34101-34104, 2003.

[8] T. T. Hartley, C. F. Lorenzo, and H. Killory Qammer, "Chaos in a fractional order Chua's system," IEEE Transactions on Circuits and Systems I: Fundamental Theory and Applications, vol. 42, no. 8, pp. 485-490, 1995.
[9] Z.-M. Ge and C.-Y. Ou, "Chaos in a fractional order modified duffing system," Chaos, Solitons \& Fractals, vol. 34, no. 2, pp. 262-291, 2007.

[10] M. P. Aghababa and H. P. Aghababa, "The rich dynamics of fractional-order gyros applying a fractional controller," Proceedings of the Institution of Mechanical Engineers-Part I: Journal of Systems \& Control Engineering, vol. 227, no. 7, pp. 588-601, 2013.

[11] M. P. Aghababa, "Chaos in a fractional-order micro-electromechanical resonator and its suppression," Chinese Physics B, vol. 21, no. 10, Article ID 100505, 2012.

[12] W. Xie, C. Wang, and H. Lin, "A fractional-order multistable locally active memristor and its chaotic system with transient transition, state jump," Nonlinear Dynamics, vol. 104, no. 4, pp. 4523-4541, 2021.

[13] P. Zhou, R. Ding, and Y.-X. Cao, "Multi drive-one response synchronization for fractional-order chaotic systems," Nonlinear Dynamics, vol. 70, no. 2, pp. 1263-1271, 2012.

[14] P. Zhou and P. Zhu, "A practical synchronization approach for fractional-order chaotic systems," Nonlinear Dynamics, vol. 89, no. 3, pp. 1719-1726, 2017.

[15] P. Muthukumar, P. Balasubramaniam, and K. Ratnavelu, "Synchronization of a novel fractional order stretch-twist-fold (STF) flow chaotic system and its application to a new authenticated encryption scheme (AES)," Nonlinear Dynamics, vol. 77, no. 4, pp. 1547-1559, 2014.

[16] P. Zhou, Z. Yao, J. Ma, and Z. G. Zhu, “A piezoelectric sensing neuron and resonance synchronization between auditory neurons under stimulus," Chaos, Solitons \& Fractals, vol. 145, no. 9, Article ID 110751, 2021.

[17] V.-T. Pham, C. Volos, S. Jafari, and T. Kapitaniak, "Coexistence of hidden chaotic attractors in a novel no-equilibrium system," Nonlinear Dynamics, vol. 87, no. 3, pp. 2001-2010, 2017.

[18] C. K. Volos, A. Akgul, V. T. Pham, and M. S. Baptista, "Antimonotonicity, crisis and multiple attractors in a simple memristive circuit," Journal of Circuits, Systems, and Computers, vol. 27, no. 2, Article ID 1850026, 2018.

[19] X. K. Hu and P. Zhou, "Circuit realization of a 3D multistability chaotic system and its synchronization via linear resistor and linear capacitor in parallel coupling," Complexity, vol. 2020, Article ID 9846934, 10 pages, 2020.

[20] Z. Liu, J. Ma, G. Zhang, and Y. Zhang, "Synchronization control between two Chua's circuits via capacitive coupling," Applied Mathematics and Computation, vol. 360, pp. 94-106, 2019.

[21] Z. Liu, C. Wang, W. Jin, and J. Ma, "Capacitor coupling induces synchronization between neural circuits," Nonlinear Dynamics, vol. 97, no. 4, pp. 2661-2673, 2019.

[22] Z. Yao, J. Ma, Y. Yao, and C. Wang, "Synchronization realization between two nonlinear circuits via an induction coil coupling," Nonlinear Dynamics, vol. 96, no. 1, pp. 205-217, 2019.

[23] J. Ma, F. Wu, A. Alsaedi, and J. Tang, "Crack synchronization of chaotic circuits under field coupling," Nonlinear Dynamics, vol. 93, no. 4, pp. 2057-2069, 2018.

[24] H. Y. Jia, Z. Q. Chen, and W. Xue, "Analysis and circuit implementation for the fractional-order Lorenz system," Acta Physica Sinica, vol. 62, no. 14, Article ID 140503, 2013.

[25] W. Zhang, S. Zhou, X. Liao, H. Mai, and K. Xiao, "Estimate the largest Lyapunov exponent of fractional-order systems," in Proceedings of the 2008 International Conference on Communications, Circuits and Systems, pp. 1121-1124, Shanghai, China, May 2008. 
[26] P. Zhou and K. Huang, "A new 4-D non-equilibrium fractional-order chaotic system and its circuit implementation," Communications in Nonlinear Science and Numerical Simulation, vol. 19, no. 6, pp. 2005-2011, 2014.

[27] P. Zhou, X. F. Cheng, and N. Y. Zhang, "One new fractionalorder chaos system and its circuit simulation by electronic workbench," Chinese Physics B, vol. 17, no. 9, pp. 3252-3257, 2008.

[28] Z. Xu and C. X. Liu, "Realization of fractional-order Liu chaotic system by a new circuit unit," Chinese Physics B, vol. 17, no. 11, pp. 4033-4038, 2008.

[29] K. H. Sun, J. L. Yang, J. F. Ding, and L. Y. Sheng, "Circuit design and implementation of Lorenz chaotic system with one parameter," Acta Physica Sinica, vol. 59, no. 12, pp. 8385-8392, 2010. 